\title{
A Curved Spiral Antenna Above a Conducting Cylinder
}

\author{
Hisamatsu Nakano, Fellow, IEEE, and Kazuo Nakayama, Member, IEEE
}

\begin{abstract}
A curved spiral antenna above a finite hollow conducting cylinder is analyzed using the method of moments. The effects of cylinder length $2 H$ and cylinder radius $r_{c y}$ on the radiation characteristics of the spiral are evaluated. As $2 H$ increases, the cross-polarization component of the radiation field in the broadside direction decreases to a constant value (approximately $-18 \mathrm{~dB})$. When $2 H$ is greater than one wavelength $\left(\lambda_{0}\right)$, the input impedance of the spiral above a cylinder of radius $r_{c y}=0.25 \lambda_{0}$ is almost constant $(250-j 20 \Omega)$ with a gain of approximately 7 dB. The spiral above a cylinder of $\left(2 H, r_{c y}\right)=\left(2.7 \lambda_{0}, 0.25 \lambda_{0}\right)$ shows a 3-dB axial ratio bandwidth of approximately $23 \%$, which is wider than a flat spiral antenna above a flat ground plane of infinite extent.
\end{abstract}

Index Terms-Measurements, moment-method solutions, spiral antenna.

\section{INTRODUCTION}

A CURVED radiation element mounted above a conducting cylinder has been receiving attention as a mobile communication antenna. Ashkenazy et al. [1] have revealed the radiation field from a microstrip antenna above a cylinder using an assumed surface-current distribution on the antenna. Nakatani et al. [2] have obtained Green's functions in dyadic form for a dipole printed on a grounded cylindrical substrate. Habashy et al. [3] have formulated the current distribution of a patch above a cylinder using a cylindrically stratified medium approach. More recently, Rostan et al. [4] have analyzed a microstrip patch array above a cylinder using a sinusoidal current distribution on the resonant patch elements. These antennas radiate a linearly polarized wave and have a conducting cylinder of infinite length.

An antenna having circular polarization $(\mathrm{CP})$ is also useful as a mobile communication antenna [5]. A spiral antenna is a $\mathrm{CP}$ radiator and is a candidate for such an application. This paper presents a curved square spiral antenna above a conducting cylinder of finite length. It should be emphasized that the surface of the spiral antenna is parallel to the conducting cylinder.

For numerical analysis, the cylinder of finite length is approximated by grid wires [6]. The method of moments [7] is applied to an electric field integral equation [8] to obtain the current distribution on the spiral and cylinder. The radiation characteristics, including the input impedance, radiation pattern, axial ratio, and gain, are evaluated using the obtained current distributions. These radiation characteristics are compared with those of a flat spiral antenna above a flat

Manuscript received September 4, 1997; revised August 25, 1998.

The authors are with the College of Engineering, Hosei University, Koganei, Tokyo, 184-8584 Japan.

Publisher Item Identifier S 0018-926X(99)02205-X

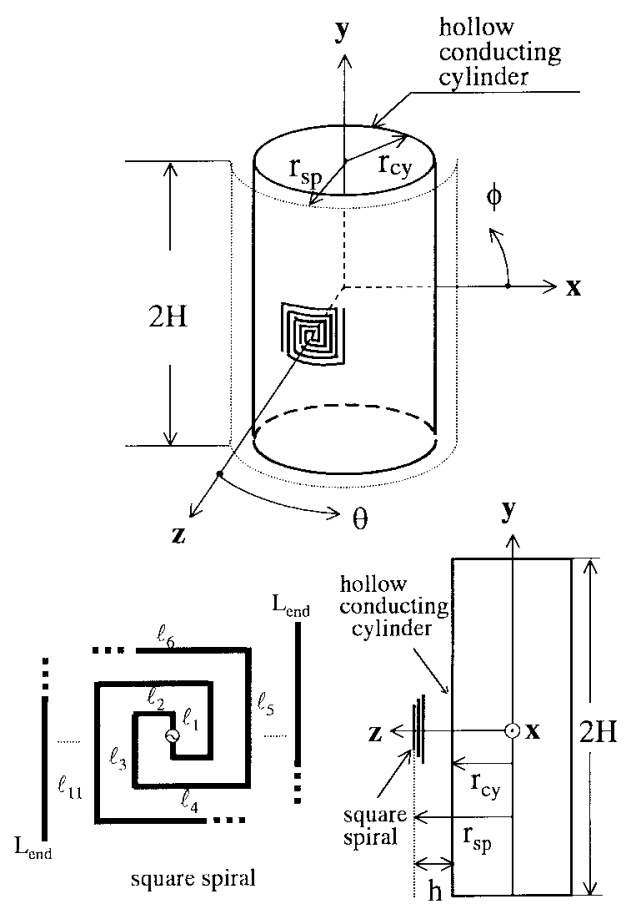

Fig. 1. Configuration and coordinate system.

conducting plate (reflector). This flat spiral is used as the reference antenna throughout this paper.

Attention is paid to investigating the effects of the cylinder length and radius on the radiation characteristics of the curved spiral. The radiation characteristics as a function of cylinder length are calculated for a fixed cylinder radius of $r_{c y}=0.25 \lambda_{0}$, where $\lambda_{0}$ is the wavelength at a test frequency of $3 \mathrm{GHz}$. In addition, the radiation characteristics as a function of cylinder radius are calculated for a fixed cylinder length of $2 \boldsymbol{H}=2.7 \lambda_{0}$. Based on these investigations, the frequency responses of the input impedance, axial ratio, and gain of a curved spiral antenna above a cylinder of $\left(2 \boldsymbol{H}, r_{c y}\right)=\left(2.7 \lambda_{0}, 0.25 \lambda_{0}\right)$ are analyzed. It is found that the curved spiral has a bandwidth of approximately $23 \%$ for a 3-dB axial ratio criterion.

Some experiments for the curved spiral are performed to confirm the validity of the theoretical results. It is found that good agreement between experimental and theoretical results is obtained.

\section{CONFIGURATION}

Fig. 1 shows the configuration and coordinate system of a curved square spiral antenna above a finite hollow cylinder whose length is designated as $2 \boldsymbol{H}$. Note that the hollow 
cylinder does not have any end caps. The spiral is located at height $h=r_{s p}-r_{c y}$, where $r_{s p}$ is the radius of the surface of the curved spiral and $r_{c y}$ is the radius of the conducting cylinder. The conducting cylinder acts as a reflector for the curved spiral.

The spiral, which is supported by air honeycomb of relative permittivity $\varepsilon_{r}=1$, is excited by a voltage source at the spiral center. Throughout this paper, the following configuration parameters are fixed: wire radius of the spiral $\rho=0.3 \mathrm{~mm}=$ $0.003 \lambda_{0}$, antenna height $h=20 \mathrm{~mm}=0.2 \lambda_{0}$, and filament lengths of the spiral $\ell_{n}=2 l_{1}(n-1)$ for $n=2,3,4, \cdots, 10$ with a start filament length of $\ell_{1}=1.7 \mathrm{~mm}=0.017 \lambda_{0}$ and an end filament length of $\ell_{11}=30.8 \mathrm{~mm}=0.308 \lambda_{0}$. The cylinder radius $r_{c y}$, the spiral radius $r_{s p}$, and the cylinder length $2 \boldsymbol{H}$ are varied subject to the objectives of the analysis.

A flat spiral antenna $\left(r_{s p}=\infty\right)$ with the above-mentioned configuration parameters, backed by a flat conducting reflector of infinite extent $\left(r_{c y}=\infty, 2 \boldsymbol{H}=\infty\right)$, radiates a circularly polarized wave with an axial ratio of $1.4 \mathrm{~dB}$. (Note that the height $\left(r_{s p}-r_{c y}\right)$ is held constant while $r_{s p}$ and $r_{c y}$ tend to infinity, and this height is the same as that for the finite-radius cylinder case.) This flat spiral is used as the reference antenna in this paper.

\section{NUMERICAL RESULTS}

The cylinder is approximated by grid wires, which are uniformly spaced in the vertical and circumferential directions. The currents along the spiral and cylinder are obtained using the method of moments in which piecewise sinusoidal expansion and weighting functions [8] are used. The radiation characteristics, including the input impedance, radiation pattern, axial ratio, and gain, are evaluated on the basis of the obtained current distribution. Throughout this paper the axial ratio is always evaluated on the $z$ axis (broadside).

A preliminary investigation shows that the gain converges as the grid-wire spacing decreases. From the convergence test of the gain, a grid-wire space of approximately $0.06 \lambda_{0}$ is used in the following analysis [9]. Note that the radius of the grid wires is chosen to be the same as that of the spiral arm wire $\left(0.003 \lambda_{0}\right)$.

\section{A. Effects of Cylinder Length $2 \boldsymbol{H}$}

First, we investigate the effects of the cylinder length $2 \mathrm{H}$ on the radiation characteristics, fixing the cylinder radius to be one-quarter wavelength $\left(r_{c y}=0.25 \lambda_{0}\right)$ with a spiral radius of $r_{s p}=r_{c y}+h=0.25 \lambda_{0}+0.2 \lambda_{0}$.

Fig. 2 shows the input impedance $Z_{\text {in }}=R_{\text {in }}+j X_{\text {in }}$ as a function of the cylinder length $2 \boldsymbol{H}$. It is found that the input impedance is almost constant when the cylinder length is greater than one wavelength $\left(\lambda_{0}\right)$. The resistance value is approximately $250 \Omega$ with a reactance value of approximately $-20 \Omega$.

The behavior of the input impedance is related to the current distribution along the spiral arms. Fig. 3 shows the current distribution $I=I_{r}+j I_{i}$ for $2 \boldsymbol{H}=2.7 \lambda_{0}$. Detailed calculations reveal that the spiral has a gradually decaying traveling-wave current with an almost constant value at the feed point when

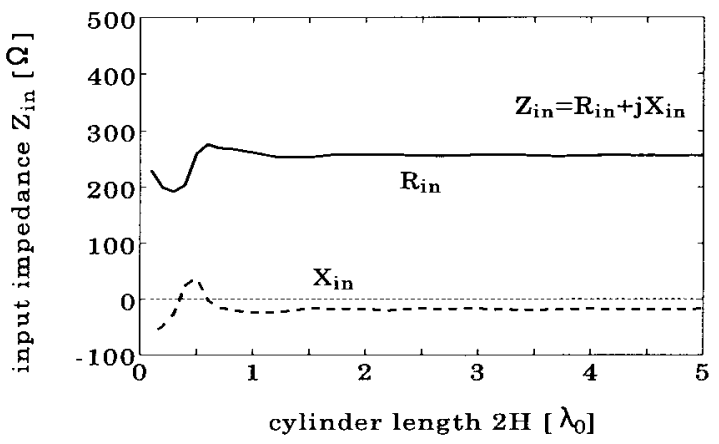

Fig. 2. Input impedance as a function of cylinder length $2 \boldsymbol{H} . r_{c y}=0.25 \lambda_{0}$, $r_{s p}=0.25 \lambda_{0}+0.2 \lambda_{0}$.

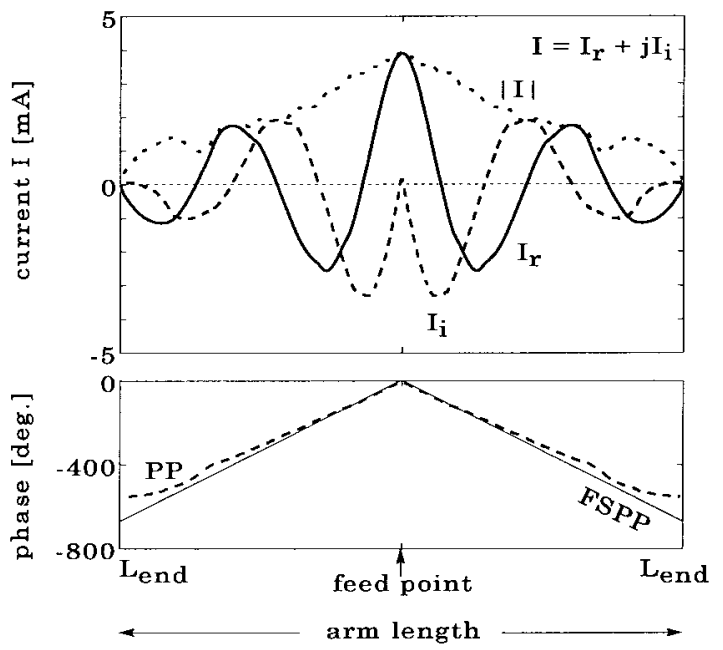

Fig. 3. Current distribution on spiral arms. $r_{s p}=0.25 \lambda_{0}+0.2 \lambda_{0}$, PP: phase progression - - -, FSPP: free-space phase progression

the cylinder length $2 \boldsymbol{H}$ is greater than $\lambda_{0}$. This leads to a constant input impedance.

Regarding the current distribution on the cylinder, the current (amplitude $|I|$ ) along the longitudinal grid wire at $(x, y, z)=\left(0, y, 0.25 \lambda_{0}\right)$ (one of the grid wires modeling the cylinder) is shown in Fig. 4, where the cylinder length $2 \boldsymbol{H}$ ranges from $0.6 \lambda_{0}$ to $4.7 \lambda_{0}$. The arrows (from $y=-0.15 \lambda_{0}$ to $y=+0.15 \lambda_{0}$ ) on the cylinder illustrate the projection of the spiral into the cylinder surface. It is found that the induced current on the cylinder is concentrated under the spiral.

The radiation field is obtained as the superposition of the radiation due to the current along the spiral arms and the radiation due to the current induced along the cylinder. Fig. 5 shows the radiation patterns for different cylinder lengths. $E_{R}$ and $E_{L}$ in this figure are the right- and left-hand circularly polarized wave components, respectively.

As seen from Fig. 5, the cross-polarization component $\left(E_{L}\right)$ in the $+z$ direction decreases as the cylinder length $2 \boldsymbol{H}$ increases. The cross-polarization component is approximately $-18 \mathrm{~dB}$ when $2 H>2 \lambda_{0}$. It is also found that as the cylinder length increases, the ratio of the front lobe to the back lobe (FB ratio) of the copolarization component $E_{R}$ improves. The FB ratio is approximately $15 \mathrm{~dB}$ when $2 \boldsymbol{H}>2 \lambda_{0}$. The experimental results at a test frequency $f_{0}=3 \mathrm{GHz}$ 


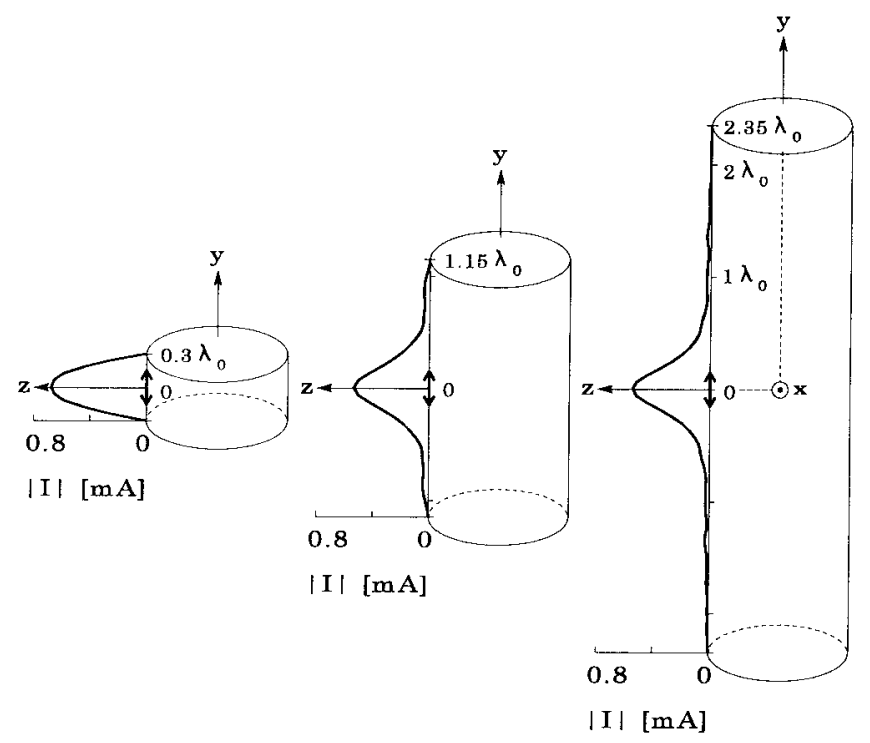

(a)

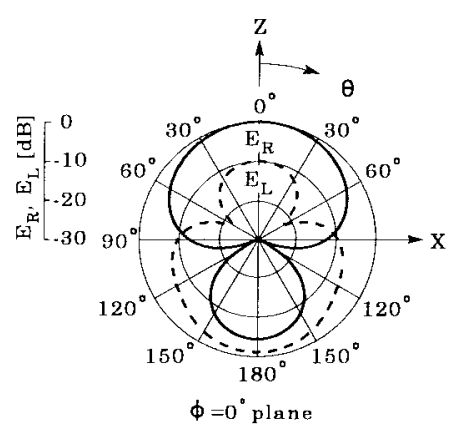

(a)
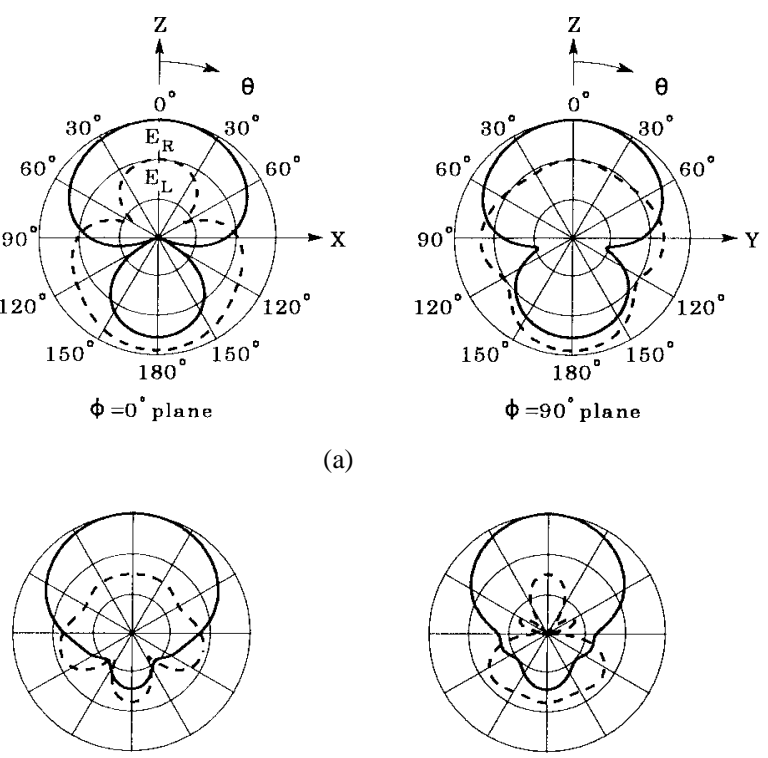

(b)
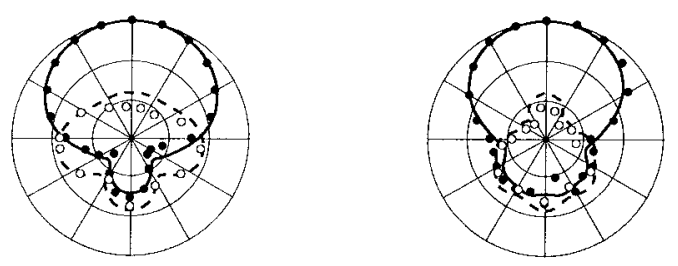

(c)
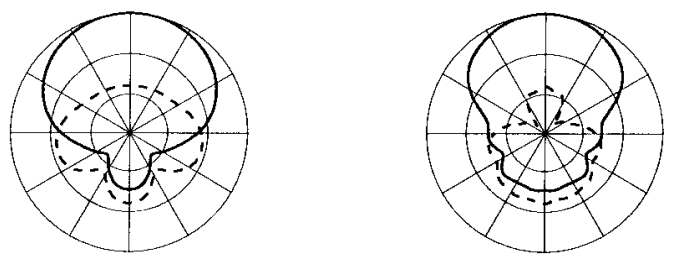

(d)

Fig. 5. Radiation pattern as a function of cylinder length $2 H . r_{c y}=0.25 \lambda_{0}$, $r_{s p}=0.25 \lambda_{0}+0.2 \lambda_{0}$

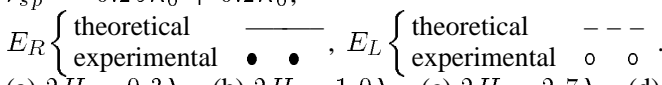

(a) $2 H=0.3 \lambda_{0}$. (b) $2 H=1.0 \lambda_{0}$. (c) $2 H=2.7 \lambda_{0}$. (d) $2 H=5.0 \lambda_{0}$.

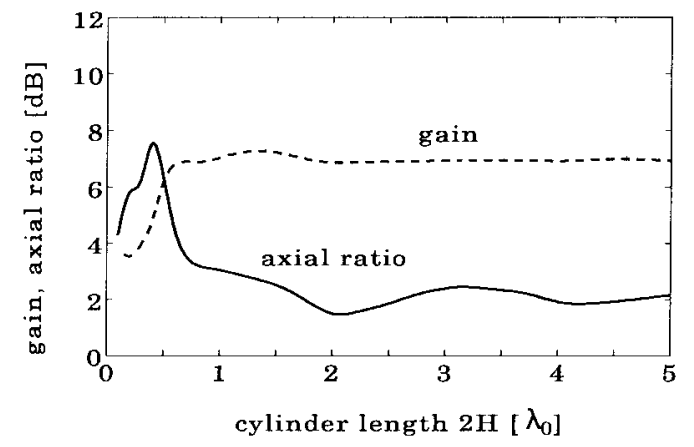

Fig. 6. Gain and axial ratio as a function of cylinder length $2 \boldsymbol{H}$. $r_{c y}=0.25 \lambda_{0}, r_{s p}=0.25 \lambda_{0}+0.2 \lambda_{0}$.

for the reference antenna, respectively. The radiation from the curved spiral is circularly polarized with an axial ratio of less than $3 \mathrm{~dB}$ for $r_{c y}>0.2 \lambda_{0}$. The value of the axial ratio 


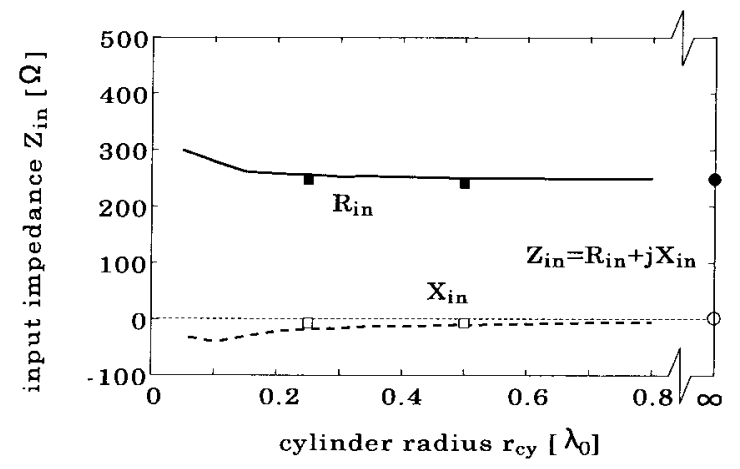

Fig. 7. Input impedance as a function of cylinder radius $r_{c y}$. $r_{s p}=r_{c y}+0.2 \lambda_{0}, 2 \boldsymbol{H}=2.7 \lambda_{0}$

$R_{\text {in }}\left\{\begin{array}{l}\text { theoretical } \\ \text { experimental }\end{array}, X_{\text {in }} \begin{cases}\text { theoretical } & - \\ \text { experimental } & \square\end{cases}\right.$ reference antenna theoretical $\left\{\begin{array}{ll}R_{\text {in }} & \bullet \\ X_{\text {in }} & \circ\end{array}\right.$.

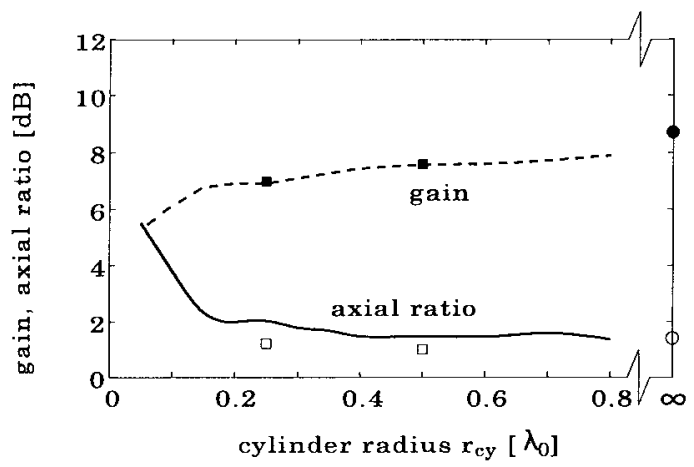

Fig. 8. Gain and axial ratio as a function of cylinder radius $r_{c y}$. $r_{s p}=r_{c y}+0.2 \lambda_{0}, 2 H=2.7 \lambda_{0}$,

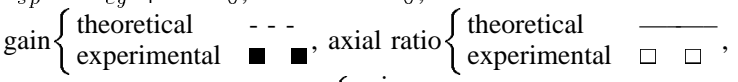
reference antenna theoretical $\left\{\begin{array}{ll}\text { gain } & \bullet \\ \text { axial ratio } & \circ\end{array}\right.$.

at $r_{c y}=0.8 \lambda_{0}$ is almost the same as that of the reference antenna.

The current (amplitude $|I|$ ) flowing along the rear half of the cylinder (from $\theta=90-270^{\circ}$ ) decreases as the cylinder radius $r_{c y}$ increases. This is shown in Fig. 9. Note that the current in this figure is calculated for the circumferential grid wire of the cylinder model at $y=0$. The decrease in the current over a region from $\theta=90-270^{\circ}$ results in less radiation from the rear region, as shown in Fig. 10, and the copolarization component $E_{R}$ has a FB ratio of more than $20 \mathrm{~dB}$ for $r_{c y}>0.5 \lambda_{0}$. The theoretical and experimental results are in good agreement in Fig. 10(b) and (d).

\section{Frequency Responses}

Effects of the cylinder length $2 \boldsymbol{H}$ and the cylinder radius $r_{c y}$ on the radiation characteristics at a frequency $f_{0}=3 \mathrm{GHz}$ were discussed in Section III-A and B. In this section, we reveal the frequency response of the radiation characteristics.

The cylinder radius $r_{c y}$ for the curved spiral antenna is chosen to be small in order to clarify the difference in the frequency responses between the curved and reference antennas: $r_{c y}=0.25 \lambda_{0}$ with cylinder length $2 \boldsymbol{H}=2.7 \lambda_{0}$,

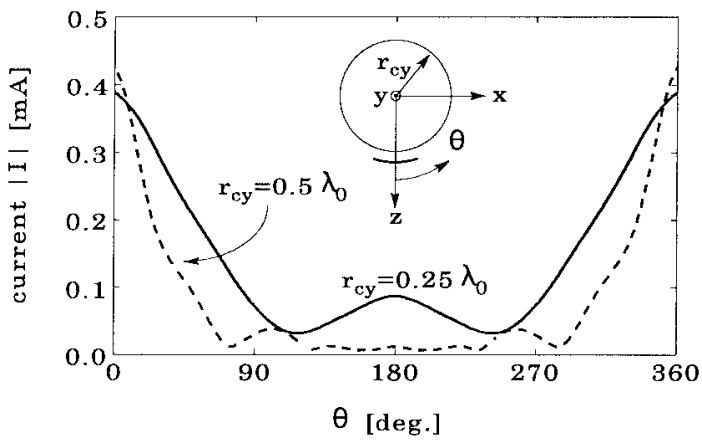

Fig. 9. Current distribution along the wire at $y=0$, which is one of the circumferential grid wires modeling the cylinder with $r_{s p}=r_{c y}+0.2 \lambda_{0}$ and $2 H=2.7 \lambda_{0}$.
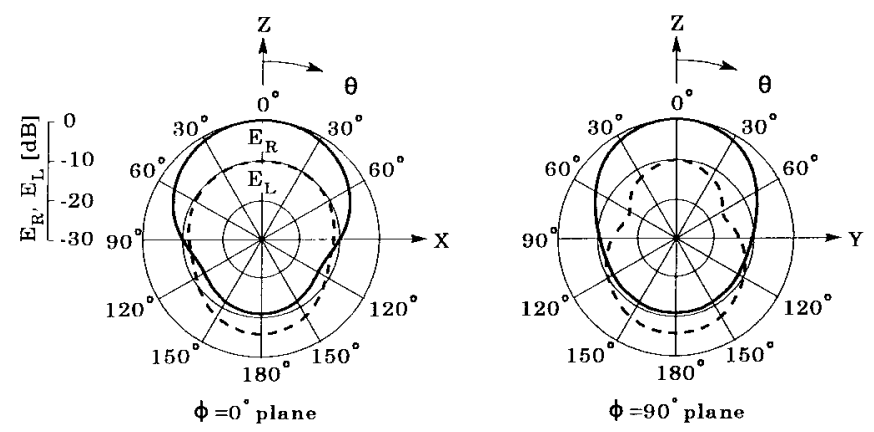

(a)
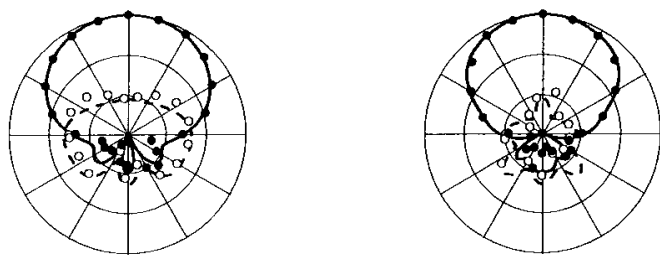

(b)
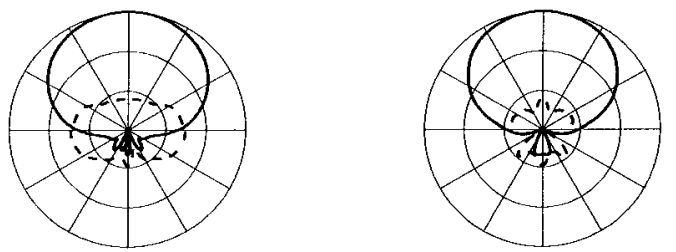

(c)
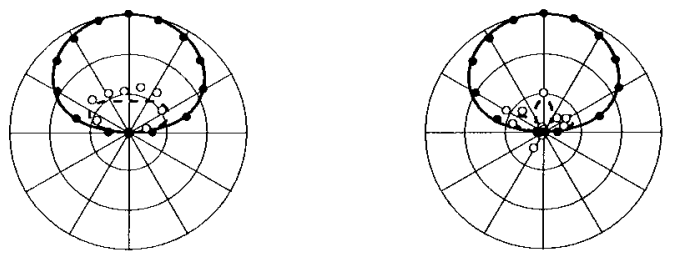

(d)

Fig. 10. Radiation pattern as a function of cylinder radius $r_{c y}$. $r_{s p}=r_{c y}+0.2 \lambda_{0}, 2 H=2.7 \lambda_{0}$,

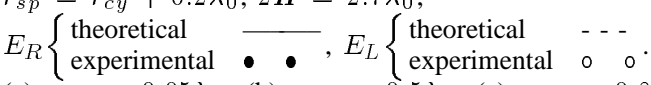

(a) $r_{c y}=0.05 \lambda_{0}$. (b) $r_{c y}=0.5 \lambda_{0}$. (c) $r_{c y}=0.8 \lambda_{0}$. (d) $r_{c y}=\infty$ (reference antenna).

where $\lambda_{0}$ is the wavelength at the frequency $f_{0}=3 \mathrm{GHz}$. The solid lines in Fig. 11 show the frequency response of the input impedance for the curved spiral. The resistance and reactance 


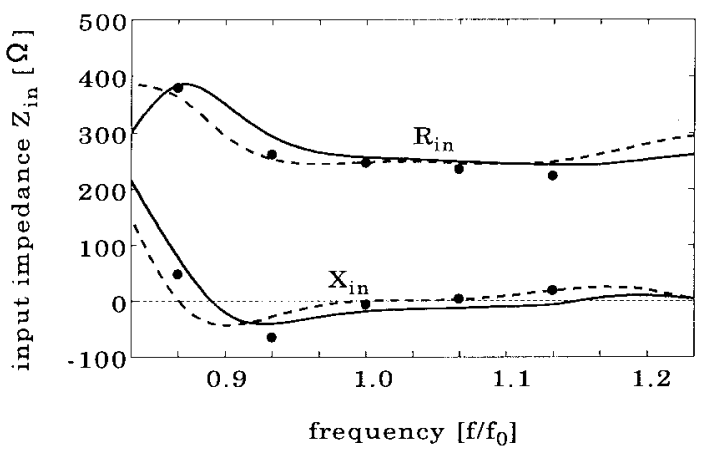

Fig. 11. Frequency response of input impedance. $r_{c y}=0.25 \lambda_{0}$, $r_{s p}=0.25 \lambda_{0}+0.2 \lambda_{0}, 2 H=2.7 \lambda_{0}$,

curved spiral antenna $\left\{\begin{array}{l}\text { theoretical } \\ \text { experimental }\end{array}\right.$

reference antenna theoretical - - -

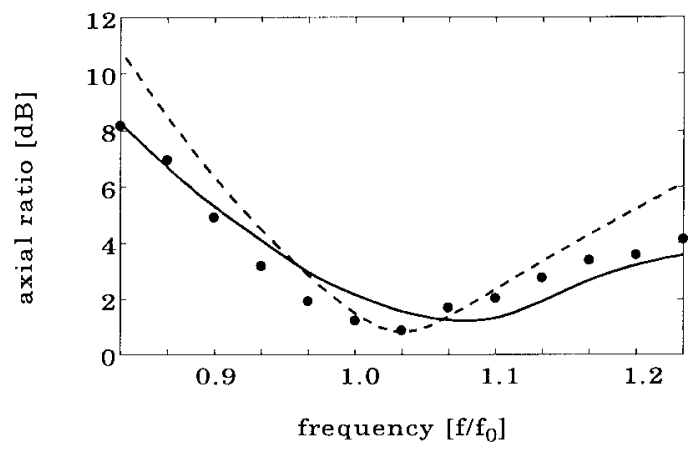

Fig. 12. Frequency response of axial ratio. $r_{c y}=0.25 \lambda_{0}$, $r_{s p}=0.25 \lambda_{0}+0.2 \lambda_{0}, 2 H=2.7 \lambda_{0}$,

curved spiral antenna $\left\{\begin{array}{l}\text { theoretical } \\ \text { experimental } \bullet\end{array}\right.$

reference antenna theoretical - - -

values for the reference antenna are shown by dashed lines. It is found that the difference in the impedance between the two antennas is not significant at frequencies higher than $f_{0}$. The curved and reference spirals have an almost pure resistance of $250 \Omega$. Note that the black dots show the experimental results for the curved spiral.

Fig. 12 shows the frequency response of the axial ratio. The curved spiral has a wider 3-dB axial ratio bandwidth than the reference antenna: $23 \%$ for the curved spiral and $15 \%$ for the reference antenna. Over these frequency bandwidths, the gains for the curved spiral and reference antenna show almost constant values (as shown in Fig. 13): approximately $7 \mathrm{~dB}$ for the curved spiral and approximately $8 \mathrm{~dB}$ for the reference antenna. The experimental results for the curved spiral are presented in Figs. 12 and 13, showing good agreement with theoretical results.

Based on the above results, it can be said that the curved spiral has wide-band performance comparable to that of the reference antenna.

\section{CONCLUSIONS}

A curved spiral antenna above a conducting cylinder of finite length is analyzed using the method of moments. A flat spiral antenna backed by a flat reflector of infinite extent is also analyzed as a reference antenna.

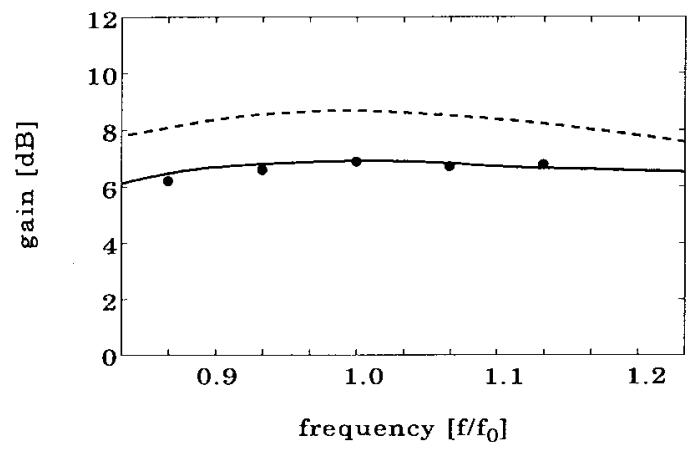

Fig. 13. Frequency response of gain. $r_{c y}=0.25 \lambda_{0}, r_{s p}=0.25 \lambda_{0}+0.2 \lambda_{0}$, $2 \boldsymbol{H}=2.7 \lambda_{0}$, curved spiral antenna $\left\{\begin{array}{l}\text { theoretical } \\ \text { experimental }\end{array}\right.$ reference antenna theoretical - - -

First, the effects of cylinder length $2 \mathrm{H}$ on the radiation characteristics are evaluated for a fixed cylinder radius of $r_{c y}=0.25 \lambda_{0}$. It is found that the curved spiral antenna has a constant input impedance $(250-j 20 \Omega)$ for cylinder length $2 \boldsymbol{H}>\lambda_{0}$ due to a constant traveling-wave current distribution along the spiral arms. As the cylinder length $2 \boldsymbol{H}$ increases, the cross-polarization component in the broadside direction decreases to a constant value (approximately $-18 \mathrm{~dB}$ ) and the FB ratio improves. The gain of the curved spiral is calculated to be approximately $7 \mathrm{~dB}$ for $2 \boldsymbol{H}>2 \lambda_{0}$, which is slightly lower than the gain of the reference antenna $\left(G_{\text {ref }}=8.7 \mathrm{~dB}\right)$.

Second, the effects of the cylinder radius $r_{c y}$ on the radiation characteristics are investigated using a curved spiral antenna with a fixed cylinder length of $2 \boldsymbol{H}=2.7 \lambda_{0}$. When $r_{c y}>$ $0.8 \lambda_{0}$, the input impedance and the axial ratio are nearly equal to those of the reference antenna. The copolarization component of the radiation field has a FB ratio of more than $20 \mathrm{~dB}$ for $r_{c y}>0.5 \lambda_{0}$.

Finally, the frequency responses of the radiation characteristics are revealed for a cylinder of $\left(2 H, r_{c y}\right)=$ $\left(2.7 \lambda_{0}, 0.25 \lambda_{0}\right)$. The curved spiral has a frequency bandwidth of $23 \%$ for a $3-\mathrm{dB}$ axial ratio criterion, which is wider than the reference antenna $(15 \%)$. It is also found that the gain of the curved spiral is approximately $7 \mathrm{~dB}$ over this $23 \%$ frequency bandwidth.

\section{ACKNOWLEDGMENT}

The authors would like to thank V. Shkawrytko for his kind discussions on the topic of this article.

\section{REFERENCES}

[1] J. Ashkenazy, S. Shtrikman, and D. Treves, "Electric surface current model for the analysis of microstrip antennas on cylindrical bodies," IEEE Trans. Antennas Propagat., vol. AP-33, pp. 295-300, Mar. 1985.

[2] A. Nakatani, N. G. Alexopoulos, N. K. Uzunoglu, and P. L. E. Uslenghi, "Accurate Green's function computation for printed circuit antennas on cylindrical substrates," Electromagn., vol. 6, no. 6, pp. 243-254, Nov./Dec. 1986.

[3] T. M. Habashy, S. M. Ali, and J. A. Kong, "Input impedance and radiation pattern of cylindrical-rectangular and wraparound antennas," IEEE Trans. Antennas Propagat., vol. 38, pp. 722-731, May 1990.

[4] F. Rostan, G. Gottwald, and W. Wiesbeck, "Design and performance of conformal microstrip patch arrays on cylindrical surfaces," in Proc. 
24th Eur. Microwave Conf. EuMC'94, Cannes, France, Sept. 1994, pp. $1756-1761$.

[5] H. Nakano, Y. Shinma, and J. Yamauchi, "A monofilar spiral antenna and its array above a ground plane-Formation of a circularly polarized tilted fan beam," IEEE Trans. Antennas Propagat., vol. 45, pp. 1506-1511, Oct. 1997.

[6] J. Moore and R. Pizer, Moment Methods in Electromagnetics. Hertfordshire, U.K.: Res. Studies, 1984.

[7] R. F. Harrington, Field Computation by Moment Method. New York: Macmillan, 1968

[8] E. Yamashita, Ed., Analysis Methods for Electromagnetic Wave Problems. Norwood, MA: Artech House, 1996, vol. 2, ch. 3.

[9] K. Nakayama and H. Nakano, "Radiation characteristics of a conformal spiral antenna," Trans. IEICE Japan, vol. J81-B-II, no. 6, pp. 609-615, June 1998 (in Japanese).

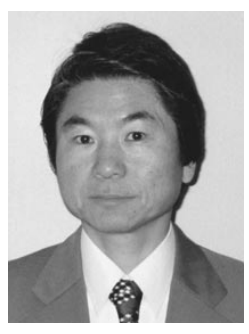

Hisamatsu Nakano (M'75-SM'87-F'92) was born in Ibaraki, Japan, on April 13, 1945. He received the B.E., M.E., and Dr.E. degrees in electrical engineering from Hosei University, Tokyo, Japan, in 1968,1970 , and 1974, respectively.

Since 1973 , he has been a member of the faculty of Hosei University where he is now a Professor in the Electronic Informatics Department. He was a Visiting Associate Professor at Syracuse University (March-September, 1981), a Visiting Professor at University of Manitoba (March-September, 1986), and a Visiting Professor at University of California, Los Angeles (September 1986-March, 1987). He developed a parabolic reflector antenna using a backfire helical feed for direct reception of broadcasting satellite (DBS) TV programs in Japan. He also developed two types of small planar DBS antennas using novel elements: curled and extremely low-profile helical elements. His other developments include microstrip antennas for global positioning systems (GPS), antennas for portable telephones, and parabolic reflector antennas for reception of communication satellite TV programs. He has published more than 150 refereed journal papers. He is the author of Helical and Spiral Antennas (Letchworth, UK: Res. Studies Press, 1987) and the coauthor of Analysis Methods of Electromagnetic Wave Problems-Vol. 2, (Norwood, MA: Artech House, 1996). His research topics include numerical methods for microwave antennas and light wave problems.

Dr. Nakano was the recipient of an International Scientific Exchange Award from the Natural Sciences and Engineering Research Council of Canada in 1986. In 1987 he received the Best Paper Award of the Institution of Electrical Engineers Fifth International Conference on Antennas and Propagation. In 1994 he received the IEEE Antennas and Propagation Society Wheeler Award.

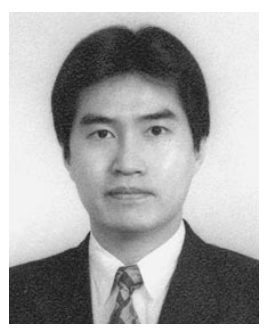

Kazuo Nakayama (M'98) was born in Hiroshima, Japan, on January 20,1966 . He received the B.E. and M.E. degrees in electrical engineering from Hosei University, Tokyo, Japan, in 1989 and 1991, respectively. He is currently working toward the $\mathrm{Ph}$.D. degree at the same university.

From 1991 to 1994, he was with the Uniden Corporation, Tokyo, Japan. His current research interests are in the design and analysis of dualpolarization antennas for satellite communication systems.

Mr. Nakayama is a member of the Institute of Electronics, Information, and Communication Engineers (IEICE) of Japan. 